\title{
Evidence for residual elastic strain in deformed natural quartz
}

\author{
Martin Kunz ${ }^{1)}$, Kai Chen ${ }^{1)}$, Nobumichi Tamura ${ }^{1)}$, Hans-Rudolf Wenk ${ }^{2}$ \\ 1) \\ Advanced Light Source, Lawrence Berkeley Laboratory, 1 Cyclotron Road, Berkeley California 94720. \\ Department of Earth and Planetary Science, University of California, Berkeley, California 94720
}

\begin{abstract}
:
Residual elastic strain in naturally deformed, quartz-containing rocks can be measured quantitatively in a petrographic thin section with high spatial resolution using Laue microdiffraction with white synchrotron x-rays. The measurements with a resolution of one micrometer allow the quantitative determination of the deviatoric strain tensor as a function of position within the crystal investigated. The observed equivalent strain values of 800-1200 microstrains represent a lower bound of the actual preserved residual strain in the rock, since the stress component perpendicular to the cut sample surface plane is released. The measured equivalent strain translates into an equivalent stress in the order of $\sim 50 \mathrm{MPa}$.
\end{abstract}

Key words: Quartz, lattice strain, residual stress, Laue microdiffraction

Introduction Quartz is ubiquitous in a wide variety of sedimentary, igneous and metamorphic rocks. It is well known for its 'undulatory' extinction when viewed with a petrographic microscope and 
crossed polarizers. This is caused by fragmentation of the single crystal into individual domains, separated by dislocation walls, and occurs during plastic deformation. Since the domains are formed by the exposure to a non-hydrostatic stress field, crystals showing these optical effects may preserve some amount of residual elastic strain. This has been suggested based on a measurable degree of optical birefringence observed in nominally uniaxial quartz crystals (Starkey 2000). The quantitative documentation of residual elastic strain preserved in quartz in a natural rock has been hampered by experimental difficulties. Neutron diffraction has been used to measure internal strains in metals (Daymond 2006; Hauk 1997; Noyan and Cohen 1987) and sandstones (Pintschovius et al. 2000; Darling et al. 2004). The disadvantage of using diffraction on bulk polycrystalline samples is that it averages over many orientations, thereby limiting spatial resolution. This limitation can be overcome by using a microfocused $(\sim 1 \mu \mathrm{m})$ synchrotron X-ray beam with a large energy spectrum (white beam) and recording local Laue single-crystal diffraction images with a high spatial resolution within a single grain. Laue diffraction has previously been used to assess qualitatively the defect structure of deformed quartz (Wenk 1966). By comparing derived lattice information with theoretical values for unstrained crystals yields a quantitative characterization of the orientation matrix of the specific local spot, as well as the deviatoric component of the strain tensor (Tamura et al. 2002). In this paper, we report a first application of microfocus X-ray diffraction to quantify the relative orientation and residual deviatoric strain in quartz from granite in the vicinity of a deformation zone. It appears that considerable residual strains are indeed preserved.

Experiments The sample we have chosen for this investigation is a granite adjacent to the Santa Rosa mylonite zone in Southern California that grades from ductile deformation at depth to brittle deformation (Wenk 2000; Pehl and Wenk 2004). An uncovered thin section of sample PC 89 was prepared in a standard way (30 $\mu \mathrm{m}$ thickness, mounted on a glass slide with epoxy). Quartz shows 
undulatory extinction (Fig. 1a) and for this study we selected a crystal that is surrounded by biotite, muscovite and plagioclase. Results from this moderately deformed quartz grain were then compared with a $30 \mu \mathrm{m}$ thick thin-section of a hydrothermally grown synthetic quartz crystal. Experiments were performed on beamline 12.3.2 of the Advanced Light Source of Lawrence Berkeley Laboratory. A white $(5 \mathrm{keV}<\mathrm{E}<22 \mathrm{keV}) \mathrm{X}$-ray beam is focused with a set of Kirkpatrick-Baez mirrors to a $1 \mu \mathrm{m} \times 1 \mu \mathrm{m}$ spot. The sample is placed on a high-precision stage and scanned through the X-ray beam at its focus spot. At each step a Laue diffraction pattern is collected in reflection geometry, using a Mar133 X-ray-CCD detector mounted at $90^{\circ}$ to the incident beam (Fig. 2, insert). The sample to detector distance is calibrated with a Si-crystal mounted on the thin section next to the scanned area. More details on the experimental set-up are given in Kunz et al. (2009). All Laue patterns were automatically indexed using the local software package Xmas (Tamura et al. 2003). A typical experimental image with some selected indexed reflections is shown in Figure 2. Considerable peak-splitting is evident, indicating significant misorientations and strain, even within the small diffracting volume. Two scans with different spatial resolution were recorded. The first enclosed an area of $1.475 \mathrm{~mm}$ x $0.575 \mathrm{~mm}$ with a step size of $25 \mu \mathrm{m}$. Within this area, a second more detailed scan recorded $0.27 \mathrm{~mm} \times 0.195 \mathrm{~mm}$ with $5 \mu \mathrm{m}$ step-resolution. The zoomed-in area with 2106 measurements which we are going to present here is indicated on Figure 1a. A scan is performed in a few hours, data processing takes considerably longer. In each Laue image, multiple spots were indexed using reference values for the quartz unit cell ( $a=4.921 \AA, c=5.4163 \AA$, Glinnemann et al. 1992). The misfit between the indexed patterns and the reference patterns are interpreted as residual elastic strain. Since the Laue patterns contain no information on the absolute size of the indexed unit cells, this only reveals the deviatoric part of the strain tensor, $\varepsilon^{\prime} 11, \varepsilon^{\prime} 22, \varepsilon^{\prime} 33, \varepsilon^{\prime} 12, \varepsilon^{\prime} 13, \varepsilon^{\prime} 23$. The strain tensor is given in sample coordinates with $\varepsilon^{\prime} 11$ parallel to $\mathrm{X}$, $\varepsilon^{\prime} 22$ parallel to $\mathrm{Y}$ and $\varepsilon^{\prime} 33$ 
perpendicular to the thin section. When fitting the residual strain from Laue patterns, we assume the volume-change to be zero. The results from the indexation were further analyzed with respect to grain overlap and differences in orientation between individual indexed domains. Only indexations with at least 30 matching reflections were considered to be valid. For two domains within a frame to be considered different crystals, their relative rotation around the c-axis had to be more than $0.5^{\circ}$ and the orientation of the hexagonal c-axes had to differ by more than $1^{\circ}$. The magnitude of the deviatoric strain tensor is estimated as the 'equivalent strain' defined as (Liu 2005)

$$
\varepsilon_{\text {eq }}=2 / 3 \sqrt{\frac{\left(\varepsilon_{11}^{\prime}-\varepsilon_{22}^{\prime}\right)^{2}+\left(\varepsilon_{11}^{\prime}-\varepsilon_{33}^{\prime}\right)^{2}+\left(\varepsilon_{22}^{\prime}-\varepsilon_{33}^{\prime}\right)^{2}+6\left(\varepsilon_{12}^{\prime 2}+\varepsilon_{13}^{\prime 2}+\varepsilon_{23}^{\prime 2}\right)}{2}} \text {. Eq. } 1
$$

Or in principle axis orientation:

$$
\varepsilon_{\text {oq }}=\sqrt{\frac{\varepsilon_{11}^{\prime}{ }^{2}+\varepsilon_{22}^{\prime}{ }^{2}+\varepsilon_{33}^{\prime}{ }^{2}}{3}} . \quad \text { Eq. } 2 \text {. }
$$

We use microstrains as units (i.e. $10^{-6} \mathrm{Eq}$ ).

Results The deviatoric strain tensor measured for the synthetic quartz serves as a reference to assess the instrumental contribution to the refined deviatoric strain tensor of 100 the natural sample. The magnitude of the equivalent strain is small ( $\varepsilon^{\prime}$ eq $\sim 300$ microstrains) and corresponds to instrumental noise (Fig. 3b). Figure $1 \mathrm{~b}$ shows the number of unique indexed grain domains per image for each scanned point for the zoomed-in region. Large coherent areas with only one valid, unique orientation are separated by relatively narrow rims where two distinct orientations could be indexed. No grains at all were successfully indexed in areas where the beam hit a solid or fluid inclusion. Figure 1c shows the change in orientation of the 
quartz grain over the corresponding area shown in Figure 1b. The orientation angle is depicted as the angle between the trace of the trigonal c-axis on the sample surface (arrow) and the vertical Y axis on this plane (counting clockwise). The Laue analysis establishes that the grain under consideration has its c-axis more or less parallel to the sample surface. The values within the measured area range by about $1^{\circ}$. Again we recognize coherent areas with small to no variation, separated by sharply changing boundaries. For us most significant are variations of the equivalent strains (Fig. 1d). They range from $\sim 600$ to 1900 microstrains. The distribution does not show a correlation with the orientation or the number of indexed grain-domains. When looking at the individual strain components of the strain tensor along the sample coordinate axes, one finds the Z-component (perpendicular to the thin section) to be much smaller ( average value -20 microstrains.) than the $\mathrm{X}$ - (average +300 microstrains, corresponding to tension) and Y- (average -300 microstrains, corresponding to compression) components. The values measured for the $\mathrm{Z}$ component are thus at or below the strain resolution of the method applied ( $\sim 50$ microstrains).

Discussion The variation of the direction of the c-axis is about $\sim 1^{\circ}$. This is consistent with the variation of optical extinction angle across the measured portion of the grain and with EBSD studies of undulatory quartz. Obviously, the Laue determination of the orientation (Fig. 1c) is more sensitive than the optical image (Fig. 1a). Within the zoomed in area,there is a bimodal distribution of orientations, which is highlighted in a histogram (Fig. 3a). Two peaks are separated by a $\sim 0.5^{\circ}$ misorientation. This is consistent with the interpretation of relatively undeformed subgrains separated by dislocation walls. The interpretation is further supported by comparing the distribution of number of grains indexed (Fig. 1b) with the map of the variation in orientation (Fig. 1c). It is apparent that the narrow areas where two or more different grains could be indexed in an image correlate with the domain boundaries depicted as areas 
with different subgrain orientation. In the areas of the domain boundaries, the incident $\mathrm{x}$-ray beam penetrates through both domains before being absorbed by the sample. The fact that this gives rise to two sharp and distinct Laue patterns indicates that the domains are separated by sharp domain boundaries, rather than a gradual bending. Comparing the equivalent strain determined from the freely grown, synthetic quartz with the values measured for natural quartz from granite we find considerable differences. For synthetic quartz equivalent strain is $\sim 300$ microstrains (Fig. 3b, shaded), which is the limit of the instrumental resolution. For the natural quartz in the Santa Rosa granite, average equivalent strains are $\sim 1000$ microstrains. The strain distribution is 400 microstrains FWHM. The clear difference between the naturally deformed quartz grain and the synthetic quartz single crystal indicates that the observed strains are not an artifact of the thin section preparation or the experimental method. For the natural quartz grain, strains perpendicular to the thin section are an order of magnitude smaller than in the section plane. We explain this by the apparent stress release perpendicular to the cut plane when preparing a thin section. Any observed strain perpendicular to the plane is thus mainly a result of Poisson type deformation caused by the in-plane stress. Since the volume strain is assumed to be zero on a crystal surface, the measured deviatoric strain is dominant within the X-Y-plane with a negative correlation between perpendicular strain components. Note the opposite sign of the measured strain components: tensile along $\mathrm{X}$ and compressive along $\mathrm{Y}$, respectively. The values refined for the out-of-plane $\mathrm{Z}$ components are an order of magnitude smaller than the in-plane components. They are in the limit of the strain resolution. The measured strain is not necessarily identical to the strain preserved in an un-cut bulk sample, but rather represents a lower limit given by the total strain minus the released component perpendicular to the surface. This has also been observed in metals (e.g., Hauk 1997). From the measured deviatoric strain tensor $\varepsilon^{\prime} \mathrm{kl}$ one can deduce the deviatoric stress field $\sigma^{\prime}{ }_{\mathrm{ij}}$ experienced by the quartz grain 
within its setting, applying Hooke's Law $\sigma^{\prime}{ }_{\mathrm{ij}}=\mathrm{c}_{\mathrm{ijkl}} \cdot \varepsilon^{\prime} \mathrm{kl}$. Using the elastic stiffness tensor by Ogi et al (2006) for $\alpha$-quartz, combined with the measured strain tensor, we derive a residual stress tensor corresponding to an equivalent stress of approximately $50 \mathrm{MPa}$. This is a first documentation of high residual lattice strains in natural minerals. Similar patterns are routinely observed in metals with the Laue technique (e.g., Tamura et al. 2003), from X-ray line broadening (e.g., Biermann et al. 1993) and with neutron diffraction (e.g., Daymond et al. 2000; Krawitz 2001). The advantage of this technique over the use of neutrons is the intrinsically higher spatial resolution to determine both orientation and residual strain tensor. The main limitation is the restriction to probing surface regions with a penetration depth of a few tens of microns and thus not obtaining full information about the stress state of a crystal. This can be partially overcome by utilizing a higher X-ray energy, as is available on high-energy synchrotrons such as the APS and ESRF. The so-called 3DXRD technique utilizes hard monochromatic X-rays (>50 keV) in transmission geometry. The beam size is chosen to be on the order of the grain size. Because the X-ray energy is fixed and known, full strain tensors may be obtained unambiguously for the indexed bulk grains (Jacobsen et al. 2007). The 3DXRD technique may be employed in the future to examine the residual stress states in deeply embedded neighborhoods of grains in natural mineral samples. The disadvantage of 3DXRD is its insensitivity to intragranular strain-differences and spatial resolution.

We have established that in natural quartz from moderately deformed granite significant lattice strains exist that provide at least a lower limit of stresses that this rock has undergone in its geologic history. However, we should not generalize based on a single sample and the method needs now to be applied to minerals from a wide range of environments. Also it needs to be explored if and how residual strains relate to crystal orientation. The method opens a whole range of exciting new possibility for stress analysis of materials of interest in mineral physics. 
Acknowledgements We are appreciative for access to beamline 12.3.2. of the Advanced Light Source (ALS). ALS is supported by the Director, Office of Science, Office of Basic Energy Sciences, Materials Sciences Division, of the U.S. Department of Energy under Contract DE-AC02-05CH11231 at Lawrence Berkeley National Laboratory and the University of California, Berkeley, California. The research was supported by grants from DOE (DE FG02-05ER15637) and NSF (EAR 0836402). Comments from reviewers helped improve the manuscript.

\section{References Cited}

Biermann, H., Ungar, T., Pfannenmuller, T., Hoffmann, G.. Borbely, A., and Mughrabi, 198 H. (1993) Local variations of lattice parameter and long-range internal stresses during cyclic deformation of polycrystalline copper. Acta Metallurgica et Materialia 41, 2743-2753.

Darling, T.W., TenCate, J.A., Brown, D.W., Clausen, B., and Vogel, S.C. (2004) Neutron diffraction study of the contribution of grain contacts to nonlinear stress-strain behavior. Geophysical Research Letters, 31 , L16604.

Daymond, M.R. (2006) Internal stresses in deformed crystalline aggregates. Chapter 16 in H.-R. Wenk, Ed. Neutron Scattering in Earth Sciences, Reviews in Mineralogy and Geochemistry 63, Mineralogical Society of America, Chantilly, VA, 427-434.

Glinnemann, J., King, H.E., Schulz, H., Hahn, T., La Placa, S.J., and Dacol, F. (1992) Crystal structures of the low-temperature quartz-type phases of $\mathrm{SiO}_{2}$ and $\mathrm{GeO}_{2}$ at elevated pressure. Zeitschrift für Kristallographie, $198,177-212$.

Hauk, V. (1997) Structural and Residual Stress Analysis by Nondistructive Methods. Elsevier, Amsterdam. 
Jakobsen, B., Poulsen, H.F., Lienert, U., and Pantleon, W. (2007) Direct determination of elastic strains and dislocation densities in individual subgrains in deformation structures. Acta Materialia, 55, 3421-3430.

Krawitz A.D. (2001) Introduction to Diffraction in Materials Science and Engineering. Wiley, New York. Kunz, M., Tamura, N., Chen, K., MacDowell, A.A., Celestre, R. S., Church, M.M., Fakra, S., Domning, E.E., Glossinger, J.M., Plate, D.W., Smith B.V., Warwick, T., Padmore, H.A., and Ustundag, E. (2009),Determination of lattice strain with microfocus Laue diffraction, Review of Scientific Instruments, submitted.

Liu, A.F. (2005) Mechanics and Mechanism of Fracture. ASM International, Schaumburg IL.

Noyan I.C. and Cohen J.B. (1987) Residual Stress - Measurement by Diffraction and Interpretation. Springer, Heidelberg.

Ogi, H., Ohmori, T., Nakamura, N., and Hirao, M. (2006) Elastic, anelastic, and piezoelectric coefficients of $\alpha$-quartz determined by resonance ultrasound spectroscopy. Journal of Applied Physics, 100, 053511.

Pehl, J. and Wenk, H.R. (2005) Evidence for regional Dauphiné twinning in quartz from the Santa Rosa mylonite zone in Southern California. A neutron diffraction study. Journal of Structural Geology, 27, 1741-1749.

Pintschovius, L., Prem, M., and Frischbutter, A. (2000) High-precision neutron diffraction measurements for the determination of low-level residual stresses in a sandstone. Journal of Structural Geology, 22, 1581 - 1585. Starkey, J. (2000) Bi-axial quartz as stress indicator. Journal of Structural Geology, 22, 383 - 390. Tamura, N., MacDowell, A.A., Spolenak, R. Valek, B.C., Bravman, J.C.,. Brown, W.L Celestre, R.S., Padmore, H.A., Batterman, B.V., and Patel, J.R. (2003) Scanning X ray microdiffraction with submicrometer white beam for strain/stress and orientation mapping in thin films. Journal of Synchrotron Radiation, 10, 137 $-143$. 
Tamura, N., MacDowell, A.A., Celestre, R.S., Padmore, H.A., Valek, B., Bravman, J.C., Spolenak, R., Brown, W.L., Marieb, T., Fujimoto, H., Batterman, B.W., and Patel, J.R. (2002) High spatial resolution grain orientation and strainmapping in thin films using polychromatic submicron X-ray diffraction. Applied Physics Letters, 80, $3724244-3727$.

Wenk, H.-R. (1966) Fehlbau in Quarzkristallen aus Tektoniten. Contriburions to Mineralogy and Petrology, $12,63-72$.

Wenk, H.-R. (1998) Deformation of mylonites in Palm Canyon, California, based on xenolith geometry. Journal of Structural Geology, 20, 559-571.

Figure Captions Figure 1. (a) Petrographic thin section of Santa Rosa granite displaying a quartz grain with undulatory extinction. Crossed polars. (b) Map of selected area showing the number of subgrains that were indexed from Laue patterns. The insert shows details of reflections across a subgrain boundary. (c) Map showing c-axis orientation relative to the vertical plot axis. The approximate orientation of the c-axis is shown by arrow. (d) Map of equivalent lattice strain as defined in the text (units in microstrains/1000). Figure 2. Individual Laue pattern with some selected reflections indexed. The insert shows a schematic of the experimental geometry.

Figure 3: Histograms of (a) c-axis misorientations and (b) equivalent lattice strain. In (b) a quartz single crystal (grey shaded bars) is compared with the deformed quartz from granite (unfilled bars). 


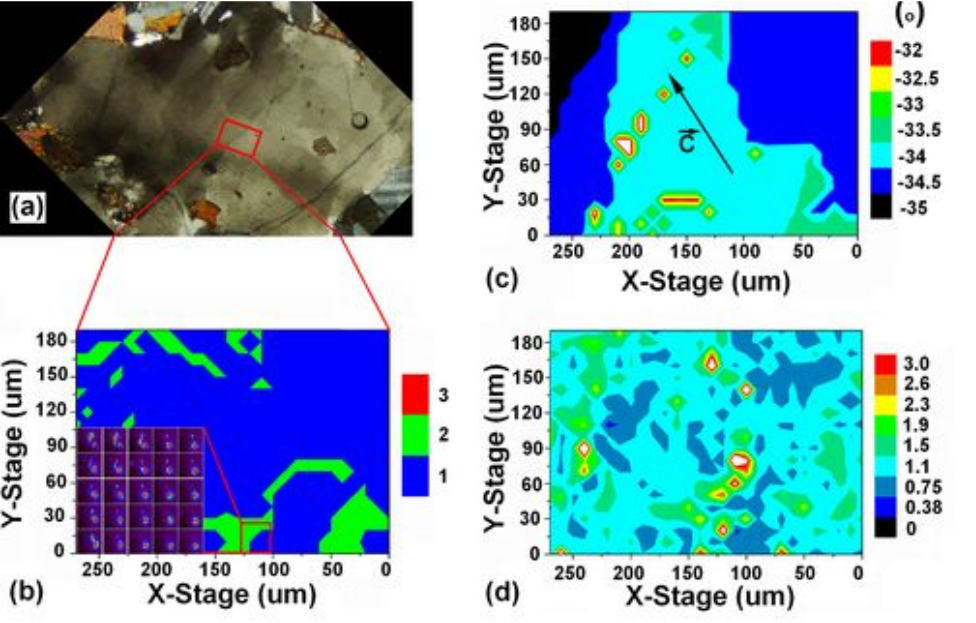




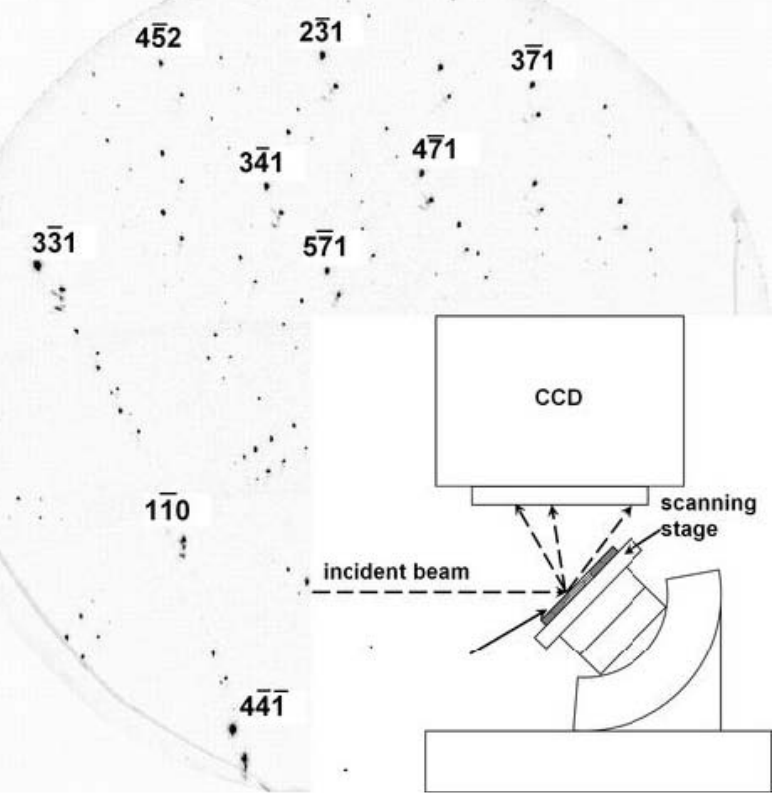




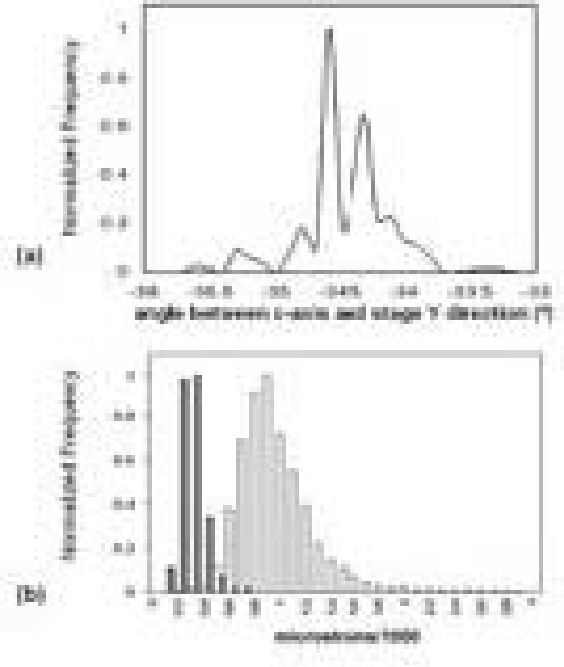

